\title{
Analysis of the Relationship between the Interaction from the Basketball to the Rebound and the Shooting Percentage
}

\author{
Fuxing Xiang* \\ Institute of P. E, Northwest University for Nationalities, Lanzhou 730030, Gansu, China
}

\begin{abstract}
Basketball in daily life is a recreational sport, he can greatly improve the work family in the quality of life of leisure time, in the movement process can not only release the pressure in your working life, can also play the role of physical exercise. The high level of basketball is a physical and technological confrontation, to win the game must improve the physical strength and basketball skills. Improve shooting is the most important means to improve basketball skills of that impact the shot hit rate of many. In this paper, the interaction of basketball and rebounding collision analysis, find a scientific method to improve the hit rate of shooting, to guide the basketball players to play a certain role in helping.
\end{abstract}

Keywords: Mechanical analysis, rebound shot, shooting percentage.

\section{INTRODUCTION}

Basketball shooting is one of the most important techniques in basketball. It is the only way to score and it is the key technology in basketball technology, and it is also the core link of basketball tactics. Whatever attack tactics is ultimately to be attributed to the shooting score, while the purpose of defense is to limit the opponent's shooting, so as to create more scoring opportunities for him. So in a sense, the basketball game is a sport that limits the opponent's score by shooting himself [1]. Therefore, the shooting rate is a critical technical indicator.

The basketball teaching and training program developed by our country in September 1987 has made clear the basketball shooting technology. From the enlightenment group (7-8 years old) to begin shooting the ball and imitate shooting exercises. Junior group B (9-11 years old), holding posture, his hands under the basket shooting, one hand under the basket shooting (moving under the basket shooting, moving one hand master shot, the road between the single hand hook shot, the road between the single hand backhand shot) and free throw teaching and training [2]. As a result, shooting is one of the key contents of the cultivation of basketball. In addition, basketball is also one of the main teaching contents of college sports department and college sports departments and colleges, and each university has a selective course of basketball, and shooting is the most favorite of the students. This shows that both teaching and training or competition, shooting is extremely important technology.

If the mechanics analysis affecting the rate of shooting factors, to improve the results of this analysis to influence for shooting skills to master the degree of feasibility and effectiveness, in order to explore increased shot rate is an effective way can be rich in basketball teaching and training of the existing theory and methods to improve the rationality of the players on the shooting process understanding, basketball training guidance, improve shooting hit rate, to guide the basketball teaching and training.

\section{THE MECHANICAL PRINCIPLES OF REBOUND SHOT}

When the elastic collision occurs in two objects, the object is in the horizontal direction of conservation of momentum, as shown in Fig. (1), on the rebound side shooting, ball trajectory as shown in Fig. (2), the backboard in shooting, ball trajectory as shown in Fig. (3).

Hoop center distance of rebound is $37.5 \mathrm{~cm}$, represented by $\mathrm{r}$, as shown in Fig. (3). $\mathrm{r}=\mathrm{h} * \tan a ́$. Because $\mathrm{r}$ is a fixed number is $37.5 \mathrm{~cm}$, when beater $\mathrm{h}$ point high, is incident angle a little, when the beater $\mathrm{h}$ point low, is incident angle a large. Both in front and side boards shooting should follow this principle [3]. Basketball shooting is the elastic collision and the ball rebounds, since the elastic collision would be in the horizontal direction of momentum conservation. In the shot, the ball is in motion, the backboard is fixed, and when the ball and rebound the elastic collision, without considering the deformation of the ball (the ball when as a particle), balls and rebound after elastic collision after running the route should be in the horizontal direction of conservation of momentum. As long as the analysis of the operation of instant ball before the collision, operation can be found after a ball. Therefore, in practice can imagine a beam is directed towards the backboard reflection from the center of the rim shot to practice shooting position of the ball (and, in some cases, the beam is to invest in the air, to make the trajectory of the ball to match reflected beam emitted from the center of the rim) and the trajectory of the beam is to practice to control the ball trajectory [4]. Ball and rebounding the moment of collision ball flight speed, angle, a point determines the ball collision trajectory, the study found. To improve the 


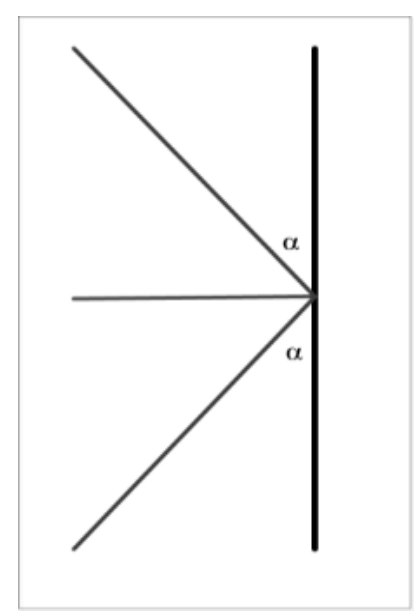

Fig. (1). Horizontal direction conservation of momentum.

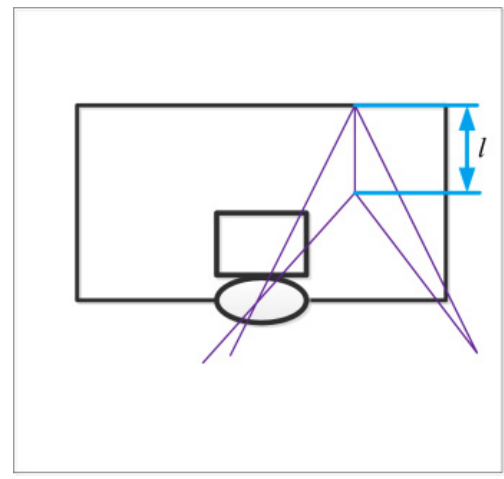

Fig. (2). Side of a plate when the ball shot trajectory.

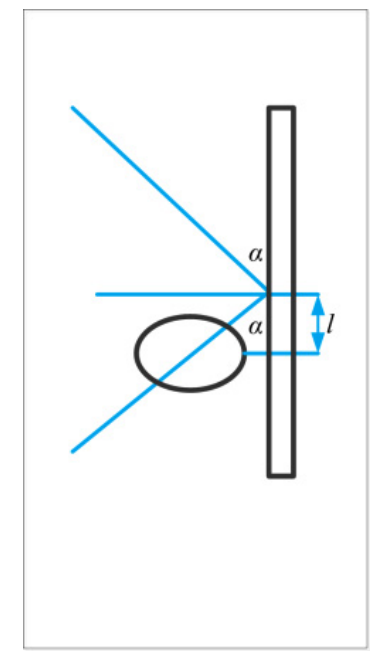

Fig. (3). A positive plate when the ball shot trajectory.

hit rate of basketball shooting must control the ball eating speed, angle, and a point.

\section{THE THEORY OF REBOUND SHOT}

\subsection{The Relationship between Ball Flight Speed and Shooting Hit Rate}

According to the $\mathrm{r}=\mathrm{h} *$ tan á, the hit rate and the speed is independent of the shooting. The ball diameter of $25 \mathrm{~cm}$, the inner diameter of the rim is $45 \mathrm{~cm}$ and hoop center dis- tance of rebound distance is $37.5 \mathrm{~cm}$ ball to in the beater is ensured by the basket, especially ball collision again basket case let the ball through the hoop, ball speed to slow down, the ball downward motion in the beater (because the momentum of the ball less than ball weight). The running track of slow speed for better control of the ball, in the horizontal direction of momentum conservation, improves the hit rate of shooting. If the fast speed of the ball, the ball in the beater will upward movement (greater than the momentum of the ball weight), unable to control the ball's trajectory, to be in the horizontal direction of conservation of momentum, making it impossible to improve the shooting hit rate.

\subsection{The Relationship between the Ball and the Back- board Collision Angle and Shooting Hit Rate}

Balls and rebounds the moment of collision angle and practice the position, the angle of different locations and rebounding are also different, the timing of the different angles ball beater is different (the ball's trajectory is divided into rising early and rising metaphase, the highest point, decrease medium and decreased at the end). Practitioners in the side of the backboard $15 \sim 45^{\circ}$ region, the practitioner is side on the backboard, the practice and rebound angle of $15 \sim 45^{\circ}$. In this region as a time for rising early, medium-term upward, the highest point, decline in the medium-term. The four stages of practice running track, easy to control the ball so, are shooting a high hit rate. Practice in rebounds positive $55 \sim 90^{\circ}$ region, practice is almost opposite the backboard, practice and rebound angle of $55 \sim 90^{\circ}$, in this area as a time only the falling stage (when the beam is to invest in the air, to make the trajectory of the ball to match emitted from the center of the rim, the reflected beam), decline at the end of practice [5]. It is very difficult to control the ball's trajectory. Therefore, shooting hit rate is low, as shown in Fig. (4).

\subsection{The Relationship between a Point and Shooting Hit Rate}

Shooting can be divided into moving shoot and fixedpoint shooting, moving shoot at the collision point can below the rim, or above the rim [6]. Beater point below the rim, be sure to raise early beater in the ball, the ball in the beater keep fast overcome the ball to the weight of the operation, and through the hoop. Fixed-point shooting the collision point must be higher than the rim a ball distance, otherwise the ball after the beater impossible through the hoop, shooting action could not be completed.

When the practitioner to determine the shooting position, the beater is a vertical line 1, the bottom line is higher than the distance of the rim of a ball point; at the top of the line is rebounding top. The distance of the line is close to the distance, and the distance is short. In the end of a line segment, the distance near the rim, large angle, a bit error is big, a shooting [7]. Along with a line segment on the move, beater from rim is becoming larger; angle becomes small beater point error gradually become smaller, and shooting hit rate decreased gradually.

\subsection{Relationship between Distance and Shooting Hit Rate}

When practitioners in the $15 \sim 45^{\circ}$ area, the ring distance is about $2 \mathrm{~m}$, a time can be increased and the highest point of 
the middle. The trajectory of the ball perfectly in the horizontal direction of momentum conservation, practice is very easy to control the ball, shooting high hit rate. Above $3 \mathrm{~m}$ distance hoop, ball beater time for decline in the mediumterm, down at the end, must let the ball in the beater instantaneous asked trajectory anastomosis trajectories in the horizontal direction of the law of conservation of momentum, the practitioners to control the trajectory of the ball has a certain degree of difficulty, the shooting hit rate is low [8].

When practitioners in the $55 \sim 90^{\circ}$ area, regardless of distance, the beater time only is decreased at the end, the practitioners difficulty of controlling the trajectory of the ball is very large, the shooting hit rate is very low.

\section{MECHANICAL ANALYSIS OF THE REBOUND SHOT}

\subsection{Throw No Spin in Cricket}

The back of the same angle is basically rebound when the ball is touched, and the angle of the basket is approximately equal to the angle of the reflector [9]. The situation of the front board and the side panel is different.

When the shot is cricket, the board point is determined by the strength and arc of the shot. The greater the arc of the ball, the greater the angle of the entry and the angle of reflection, the touch plate (in the position of the $30 \mathrm{~cm}$ ) should be correspondingly elevated. If the board is very high, and with a smaller angle into the basket hit the board, the ball will rebound in rebounds beyond the ring while flying above [10]. Investment side cricket touch, touch panel is to the same spring back angle out, if touch cricket in the from the right 45 degree angle investment and a rebound should also be 45 degrees, to make the bounce of the ball through the basket core, bumping board point should rebounds Center right $37.5 \mathrm{~cm}$ from the best. Because the boards to basket heart distance are $37.5 \mathrm{~cm}$, the reflection angle is 45 degrees, exactly constitutes a triangle, and in accordance with the requirements of the pitch of the ball hit rate is higher.

\subsection{Stress Analysis of the Positive Plate After Rotating the Ball}

After the ball in front of the board, because the ball rotation by the rebound reaction, compared with the rotation of the ball into the basket, can increase the angle, more conducive to the basket. If the ball does not rotate, the ball hit the backboard by anti $\mathrm{F}$ effect of elastic rebound, get a smaller angle into the basket, as shown in Fig. (4); because in touch after rebound, with the exception of a rebound $F$ effect, at the same time by the rotation of the rebound caused by the tangential reaction force $f$ role, so that the ball is under the action of anti-elastic $F$ and downward rebound tangential reaction force $f$. in the first, will make the spin of the ball slow down; second, the downward tangential force so that the ball rebounds adds a downward speed, so $v^{\prime}$ reflection angle speed is no longer $\mathrm{v}$, but $\mathrm{v}^{\prime}$ and $\mathrm{v}$ synthesis rate of $\mathrm{v}_{\mathrm{N}}$ [11]. The two force direction pointing the rim, the speed of synthesis $v_{i}$ direction force generated by the point to the ring. Therefore, to invest in the rotating touch the cricket, not rebound in the direction of the velocity $\mathrm{v}$ along the back, but in the backboard friction counterforce role to bounce back in the direction of the velocity $\mathrm{v}_{\mathrm{N}}$. Make the ball larger in the basket, and easily in the basket, as shown in Fig. (5). The higher the ball, the higher the rebound force, the more the ball can get into the basket angle, and it can improve the shooting rate. Pitching rotation cricket than non-rotating playing cricket flight radian to be higher, the cast positive cricket is touch the backboard of the vertical plane, because the ball is rotated backward, beater ball rotation force is to the backboard and rebounding tangential reaction force downward effect on the ball. So cast back spin on the ball, in the beater vertically falling speed increased, and reflection angle increased, infer to invest in the rotary positive cricket, beater point even slightly higher can still hit.

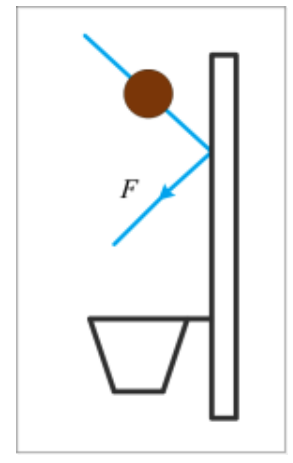

Fig. (4). Not a positive spin-sketch board.

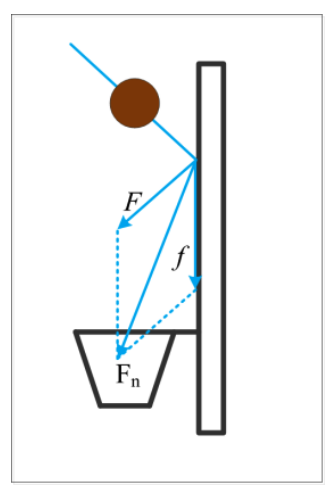

Fig. (5). After a positive spin-sketch board.

\subsection{The stress Analysis of Front Panel of Front Swing Ball}

If sphere does not rotate, touch panel, rebound force $\mathrm{F}$ acting, a bounced ball radian lower, the rebound is larger, the ball is not easy to enter the basket, as shown in Fig. (6). If the sphere pronation, in touch panel by rebound and pronation arising due to the reaction force at the same time, both the force direction is closer to the basket in vertical direction, as shown in Fig. (7), and before the ball rotating speed faster, suffered greater reaction force. The direction of the resultant force is close to in the basket ring vertical surface, can further increase the ball into the basket probability.

\subsection{The Characteristics of Side Spin Ball in Basket Shooting}

The basket area has always been the offensive and defensive battleground, the basket shot is quite powerful. Due to 


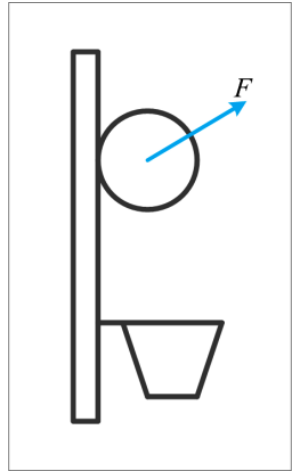

Fig. (6). Not spin the ball rebounds pop-up diagram.

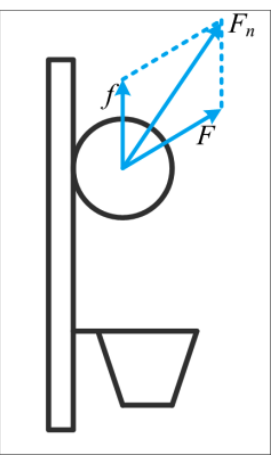

Fig. (7). Rotary ball rebounds before the pop-up diagram.

the opponent's tight defense, offensive players in the basket is very difficult to find appropriate aiming point directly to complete shooting action, more is in the basket with a completed shooting [12]. If the ball does not side spin, the rebound into the basket, a little smaller suitable. If you want the ball through the hoop in the center after the rebound, then this is the only point A, as shown in Fig. (8).

The other tight defense in the game, the offensive player to find that a point is very difficult, to compensate for this shortcoming, the ball cannot hit the A point can also be the purpose of the basket; side spin ball came into being. Under normal circumstances, in the basket on the right side of the shooting, the ball along the clockwise rotation; in the basket on the left side of the shooting, the ball according to counter clockwise rotation [13]. If the ball does not make the rotary motion, the ball hit the backboard B, the rebound cannot get into the basket of possibility; but if the sphere itself sidespin and by clockwise rotation, touch the backboard by rebound $\mathrm{F}$ and sidespin arising due to the reaction force $\mathrm{F}$ is also used, direction of the total force of the two points to the rim, so that the ball is possibility into the basket, as shown in Fig. (9). And side spin faster and rebound reaction force $F$ is greater, reflection angle is increased obviously, ball force direction will more biased towards the rim. For shooting a point ring side rotary distance, rotation speed of the ball is particularly important. On the side of rotating cast cricket, the twisting wrist rotation and fingers, causing the ball around the sagittal axis of rotation. After a ball, the reflection angle will be significantly larger than the angle into the basket [14]. Therefore, the board is also higher than the ball from the basket center farther without rotating; it is not easy to make the ball fly outside the basket. We can use this rule to improve the shooting percentage.

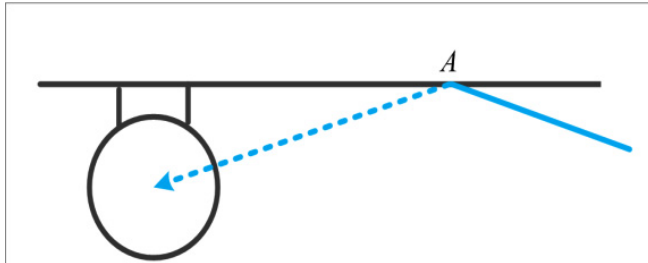

Fig. (8). Ball contingency spin a plate diagram.

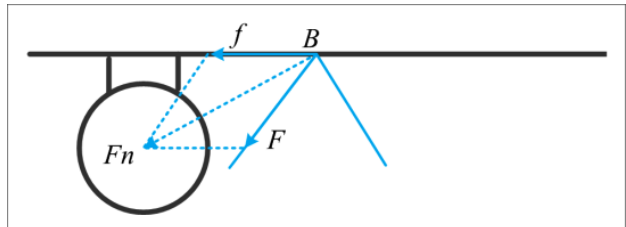

Fig. (9). Tice basket right spin dressed up for basketball sketch.

Through the above analysis that, under the basket offensive players if it is unable to find the appropriate beater, the rational use of the ball sidespin, can also get into the basket.

\section{CONCLUSION}

The from the angle of shooting analysis the relation of interaction between basketball and rebounding, and discussed under different conditions caused by different into the basket and the possibility of many impacts the shot hit rate. In the fierce competition of modern basketball games, athletes must be skilled in the complex and changeable skills and tactics and use of the technology of shooting. The front and back shots of the shots depend on the shots.. The first rotation is mainly used for moving underhand shot and posterior circumflex is mainly used for the long-range shooting; basketball rotational speed is faster, ball better stability and posterior circumflex increased arc of the ball's flight, to improve the hit rate, pronation increased ball speed, for a break shot. The shot action is made up of many technical links, and each link is a supplement to the whole [15]. Therefore, master shot technology should pay attention to the function of the technology and the coordination of the operation relationship, and pay attention to the comprehensive effect of the link technology.

It is suggested that the basketball training should take the shot technology as the center, drive the other technology to develop comprehensively, other techniques and tactics training, and also should take the creation good shot chance as the target [16]. Coaches to strict training of athletes training hard, starting from the actual, combined with technique of pass, dribble, break through and the fake action are shooting training, which is conducive to the cultivation of awareness of athletes rose to the occasion. In addition, strengthen the psychological and style training of players, but also to improve the players in the game shooting rate.

\section{CONFLICT OF INTEREST}

The author confirms that this article content has no conflict of interest.

\section{ACKNOWLEDGEMENTS}

Declared none. 


\section{REFERENCES}

[1] M. Jeličić, M. Trninić, and I. Jelaska, "Differences between three types of basketball players on the basis of situation-related efficiency,"Acta Kinesiologica, vol. 1, pp. 82-89, 2010.

[2] J. Liu, "The correlation analysis and strategy between basketball scoring index and physical fitness based on principal component model," Journal of Chemical \& Pharmaceutical Research, vol. 6, no. 3, pp. 1-25, 2014.

[3] P. Mačura, "Proposal for a didactic aid to practice the bank shots in basketball," Sport Science Review, vol. 19, no. 2, pp. 47-61, 2010.

[4] V. Ragothaman, "Psychomotor distinction in basket ball performance among state players," vol. 22, no. 6, pp. 11-19, 2012.

[5] L. Gan, "Western players in 2013 NBA all-star game based on MATLAB," Information Technology Journal, vol. 12, no. 16, pp. 3612-3618, 2013.

[6] J. Yang, and R. Wang, "Analysis of the optimal orientation measuring model in the competition ability of basketball elite athletes," Journal of Convergence Information Technology, vol. 8, no. 9, p. 405, 2013.

[7] C. Cortis, H. Tessitore, and C. Lupo, "Inter-limb coordination, strength, jump, and sprint performances following a youth men's basketball game,"The Journal of Strength \& Conditioning Research, vol. 25, no. 1, pp. 135-142, 2011.

[8] W. R. Strawser, and J. W. Strawser, "Discussing variance analysis with the performance of a basketball team,"Issues in Accounting Education, vol. 29, no. 3, pp. 481-495, 2013.
[9] C. Shih, "Aiming strategy error analysis and verification of a billiard training system," Knowledge-Based Systems, vol. 23, no. 7, pp. 732-742, 2010.

[10] C. Rodríguez-Rellán, R. F. Valcarce, and E. B. Esnaola, "Shooting out the slate: working with flaked arrowheads made on thin-layered rocks," Journal of Archaeological Science, vol. 38, no. 8, pp. 19391948, 2011.

[11] M. A. Holowchak, "The "Measure" of an athletic achievement1 character versus production, or a forced dichotomy in competitive sport,"Journal of the Philosophy of Sport, vol. 38, no. 1, pp. 88102. 2011.

[12] T. F. D. Kanthack, M. Bigliassi, and L. F. Vieira, "Acute effect of motor imagery on basketball players' free throw performance and self-efficacy," Revista Brasileira de Cineantropometria \& Desempenho Humano, vol. 16, no. 1, pp. 47-57, 2014.

[13] Shih C. Analyzing and Comparing Shot Planning Strategies and Their Effects on the Performance of an Augment Reality Based Billiard Training System. International Journal of Information Technology \& Decision Making, 2014, 13(03): 521-565.

[14] C. Shih, "Zero tolerance cue angle analysis and its effect on successive sink rate of a low cost billiard reposition control tutoring system," Knowledge-Based Systems, vol. 30, pp. 17-34, 2012.

[15] Y. Köklü, U. Alemdaroğlu, and F Koçak, "Comparison of chosen physical fitness characteristics of Turkish professional basketball players by division and playing position," Journal of Human Kinetics, vol. 30, pp. 99-106, 2011.

[16] V. Arora, G. Bhushan, and M. Aggarwal, "Static structural CAE analysis of symmetrical $65 \mathrm{Si} 7$ leaf springs in automotive vehicles," Engineering Solid Mechanics, vol. 3, no. 1, pp. 59-74, 2015.

Received: June 10, 2015

(C) Fuxing Xiang; Licensee Bentham Open.

This is an open access article licensed under the terms of the (https://creativecommons.org/licenses/by/4.0/legalcode), which permits unrestricted, noncommercial use, distribution and reproduction in any medium, provided the work is properly cited. 